\title{
Postoperative pain scores at home after adenotonsillectomy in children between 6 and 12 of age are associated with pre-existing emotional/behavioral problems
}

\author{
$\underline{\text { Snaterse Broekhuijse P.J. }}{ }^{1}$, Utens E.U. ${ }^{2}$, Poley M. ${ }^{3}$, Pirotte T. ${ }^{4}$, Weber F. ${ }^{5}$, Berghmans J. ${ }^{6}$
}

${ }^{1}$ Department of Anesthesia, ZNA Middelheim, Queen Paola Children's Hospital; Department of Anesthesiology; University Hospital Antwerp (Belgium), 2Department of Child and Adolescent Psychiatry/Psychology, Erasmus University Medical Center, Sophia Children's Hospital, University of Amsterdam-POWL/the Bascule - Rotterdam (The Netherlands), ${ }^{3}$ Institute for Medical Technology Assessment, Erasmus University Rotterdam, Department of Pediatric Surgery, Erasmus Medical Center, Sophia Children's Hospital - Rotterdam (The Netherlands), ${ }^{4}$ Department of Anesthesia, University Clinic St-Luc - Brussels (Belgium), ${ }^{5}$ Department of Anesthesia, Erasmus Medical Center Sophia Children's Hospital - Rotterdam (The Netherlands), ${ }^{6}$ Department of Anesthesia, ZNA Middelheim, Queen Paola Children's Hospital - Antwerp (Belgium); Department of Child and Adolescent Psychiatry/Psychology, Erasmus University Medical Center, Sophia Children's Hospital; Department of Anesthesia, Erasmus Medical Center Sophia Children's Hospital - Rotterdam (The Netherlands)

\section{Background and Goal of Study}

Pain at home after adenotonsillectomy is a major issue and specific child factors like pre-existing emotional/behavioral problems might be associated with higher pain scores [1]. Aims: 1. to assess the prevalence of postoperative pain at home during the first three days and at day $10 ; 2$. to test the influence of emotional/behavioral problems on postoperative pain corrected for the child's age and parental state anxiety.

\section{Materials and methods}

After IRB approval a prospective cohort study was carried out. Inclusion: eligible were all consecutive children between 6 and 12 years old, ASA $1 \& 2$, good Dutch comprehension of the parent, undergoing adenotonsillectomy in a day-care setting. Exclusion: known mental retardation.

Procedure: a standardized anesthesia procedure was performed including: 1. inhalation with sevoflurane; 2 . pain management (including Non-Steroidal Anti-Inflammatory Drugs [NSAIDs] and paracetamol). Outcome parameter: postoperative pain at home was assessed using the Parents' Postoperative Pain Measure (PPPM) during the first 3 days and at day 10.

Psychological assessment tools: 1. child: emotional/behavior problems of the child during the past 6 months were assessed using the Child Behavior Checklist (CBCL) and rated by the accompanying parent. A CBCL total problem score was obtained; 2. parent: parental state anxiety was measured by Spielberger's State-Trait Inventory (STAI). Statistics: multivariable regression analysis was carried out, using the sum of the PPPM scores at the first 3 days at home (PPPM1-3days) as the dependent variable.

\section{Results and Discussion}

A total of 57 children (mean age in months: 108.8 SD \pm 25.7 ) entered this study and $46(80 \%)$ complete cases were obtained at day 3 postoperative. During the first 3 days at home, $43.5 \%$ of children had scores $\geq 6$ on PPPM1-3days and at day 10 still $2.7 \%$ (37 complete cases). Pre-existing CBCL total problem scores were associated with higher PPPM1-3days scores (standardized regression coefficient: .373; 95\%CI [.10-.86]; P=.015), independent of the child's age and parental state anxiety. Overall the model explained $17.9 \%$ of variance (adjusted R2=.119; $\mathrm{P}=.042$ ).

\section{Conclusions}

Pre-existing CBCL emotional/behavioral problems might contribute to higher postoperative pain scores after adenotonsillectomy at home and could be helpful in identifying vulnerable children.

\section{References}

1. Dorkham M. et al. Paediatr Anaesth, 2014. 24(3): p. 239-48. 